\title{
Editorial
}

\section{Neuromuscular Disorders in Children and Adolescents}

\author{
Rudolf Korinthenberg ${ }^{1}$ \\ 1 Department of Neuropediatrics and Muscle Disorders, Center for \\ Pediatrics, University Medical Center Freiburg, Faculty of Medicine, \\ University of Freiburg, Freiburg, Germany
}

Neuropediatrics 2017;48:209-210.

The term "neuromuscular disorders" covers a large variety of diseases with the common feature of predominant affection of the neuromuscular unit including motor neuron, peripheral nerve, neuromuscular junction, or muscle fiber. In children and adolescents, most conditions are due to hereditary causes. However, the acquired, mostly inflammatory, and treatable diseases must not be missed.

Since the identification of dystrophin mutations in Duchenne muscular dystrophy in 1985 , a steadily increasing number of causative genes has been identified, and this led to improved diagnosis, prognosticating, genetic counseling, and pathophysiological understanding. However, at the same time, the sheer number of different genes and the frequent phenomenon of genes with different phenotypes, as well as common phenotypes for different genes represent an increasing challenge for clinicians and geneticists. Modern multigene panels help to solve this problem, but not unexpectedly they bring with them new problems, for example, the analysis, and interpretation of huge amounts of data.

Only during the past decade, the improved etiological understanding of these diseases gave rise to a large number of gene- or even mutation-specific animal models enabling pathophysiological and eventually therapeutic research. A large number of therapeutic principles have been developed in the preclinical domain, and some of them have already entered the stage of clinical trials or even routine care. The development of clinical trials necessitated intensive clinical research in the natural history of these diseases to be able to delineate reliable, valid, and responsive clinical and paraclinical outcome measures comprising clinical function tests, imaging, and laboratory measures.

As long as no curative therapy has been found especially for the genetic diseases, treatment has to include supportive measures, such as physiotherapy, bracing, respiratory treatment, cardiac care, and assisted feeding techniques. Along with these measures, life expectancy, for example, in Duchenne muscular dystrophy has been prolonged by more than 10 years. Again, driven by the requirements of clinical trials, it became necessary to harmonize these measures of care in the experimental groups to avoid study biases. This and the patients' needs led to the definition of "standards of care" for the most important disease groups such as Duchenne muscular dystrophy, spinal muscular dystrophy, and congenital myopathies. ${ }^{1-4}$ Faced with a lack of controlled study data in these fields, international groups of experts defined these "standards" based on the best available evidence from the literature and personal experience. Starting from scientific and clinical efforts, these standards of care can also be used in the political field to discuss and hopefully improve patient care in different countries and health systems. ${ }^{5}$

I am proud that in this special issue of Neuropediatrics, some of the most renowned national and international groups in the field of neuromuscular disorders offer their current perspectives on diagnosis and treatment, enabling the broader field of pediatric neurologists to keep up with these thrilling developments.

\section{References}

1 Bushby K, Finkel R, Birnkrant DJ, et al; DMD Care Considerations Working Group. Diagnosis and management of Duchenne muscular dystrophy, part 1: diagnosis, and pharmacological and psychosocial management. Lancet Neurol 2010;9(01):77-93

2 Bushby K, Finkel R, Birnkrant DJ, et al; DMD Care Considerations Working Group. Diagnosis and management of Duchenne muscular dystrophy, part 2: implementation of multidisciplinary care. Lancet Neurol 2010;9(02):177-189

3 Wang CH, Finkel RS, Bertini ES, et al; Participants of the International Conference on SMA Standard of Care. Consensus statement
Address for correspondence Prof. Dr. Rudolf Korinthenberg, MD, PhD, Department of Neuropediatrics and Muscle Disorders, Center for Pediatrics, University Medical Center Freiburg, Faculty of Medicine, University of Freiburg, Freiburg, Germany (e-mail: rudolf. korinthenberg@uniklinik-freiburg.de).
Issue Theme Neuromuscular Disorders in Children and Adolescents; Guest Editor: Rudolf Korinthenberg, MD.
C 2017 Georg Thieme Verlag KG Stuttgart · New York
DOI https://doi.org/ $10.1055 / \mathrm{s}-0037-1603980$. ISSN 0174-304X. 
for standard of care in spinal muscular atrophy. J Child Neurol 2007;22(08):1027-1049

4 Wang $\mathrm{CH}$, Dowling JJ, North $\mathrm{K}$, et al. Consensus statement on standard of care for congenital myopathies. J Child Neurol 2012; 27(03):363-382
5 Vry J, Gramsch K, Rodger S, et al. European cross-sectional survey of current care practices for Duchenne muscular dystrophy reveals regional and age-dependent differences. J Neuromuscul Dis 2016;3(04):517-527 